\title{
In-patient suicide: selection of people at risk, failure of protection and the possibility of causation
}

\author{
Matthew Michael Large, Daniel Thomas Chung, Michael Davidson, Mark Weiser and
}

Christopher James Ryan

\section{Background}

Being a current psychiatric in-patient is one of the strongest statistical risk factors for suicide. It is usually assumed that this strong association is not causal but is a result of the combination of the selection of high-risk patients for admission and the imperfect protection from suicide afforded by psychiatric wards. Logically, a third factor, which is causal might play a role in the association. It has recently been suggested that adverse experiences in psychiatric units such as trauma, stigma and loss of social role might precipitate some in-patient suicides.

\section{Aims}

To consider whether there is a causal association between psychiatric hospitalisation and suicide.

\section{Method}

We used the framework of Austin Bradford Hill's criteria for assessing causality in epidemiology to consider the possibility that psychiatric hospitalisation might causally contribute to the extent and variation in in-patient suicide rates.

\section{Results} suicide clearly meets five of the nine Hill's criteria
The association between psychiatric hospitalisation and

(strength of association, consistency, plausibility, coherence and analogy) and partially meets three of the remaining four criteria (gradient of exposure, temporality and experimental evidence).

\section{Conclusions}

Admission to hospital itself might play a causal role in a proportion of in-patient suicides. The safety of being in hospital with respect to suicide could be examined with a large-scale randomised controlled trial (RCT). In the absence of an RCT, the possibility of a causal role provides further impetus to calls to make care in the community more available and psychiatric hospitals more acceptable to patients.

\section{Declaration of interest}

M.M.L. and C.J.R. have provided expert testimony in legal proceedings following in-patient suicide.

\section{Copyright and usage}

(C) The Royal College of Psychiatrists 2017. This is an open access article distributed under the terms of the creative Commons Non-Commercial, No Derivatives (CC BY-NC-ND) license.

The most obvious and easily accepted reason for the high rate of Rates of suicide by current psychiatric in-patients greatly exceed those of the general community. A recent meta-analysis of studies published worldwide in the past 60 years estimated that current psychiatric in-patients (including those on approved and unapproved leave) had a pooled suicide rate of 147 suicides per 100000 in-patient years, ${ }^{1}$ a figure that is more than 12 times global population suicide rates. $^{2}$ The meta-analysis also found extraordinary variation in suicide rates between studies, with the highest reported rate of inpatient suicide being 10490 per 100000 patient years, ${ }^{3}$ which was more than a 1000 times higher than the study with the lowest rate of 9 per 100000 patient years. ${ }^{4}$ The high between-study variation in suicide rates was not confined to these two outliers: the first quartile suicide rate was 99 per 100000 per annum, the third quartile rate was 808 per 100000 per annum and the $I^{2}$ measure of between-study heterogeneity was $98 \%$ of its maximum value. This between-study variation was partially explained by a significant rise in in-patient suicide rates over recent decades $(Z=6.00, P<0.001)$. For example, the pooled rate of in-patient suicides reported worldwide between 1960 and 1979 was 68 per 100000 patient years, whereas the equivalent figure for suicides after 1999 was 646 per 100000 in-patient years, a rate of suicide that is almost 60 times the 2012 global suicide rate. ${ }^{2}$ This suggests that in-patient status might confer a higher suicide risk than any other known suicide risk factor.

When considered as a risk factor for suicide, admission to hospital is complex and multifaceted. Furthering understanding of the component factors that contribute to the strength of the association between in-patient status and suicide might assist in reducing in-patient suicide rates. psychiatric in-patient suicide is a selection effect. This explanation posits that the generally high rate of in-patient suicide is because of patient factors that lead to both admission to hospital and suicide, factors that include mental illness, suicidal behaviour, substance dependence, unemployment, marital breakdown and low socioeconomic status. Such a selection effect might also operate by the earlier discharge of lower-risk patients. Selection might account for some variation in in-patient suicide rates between studies because hospitals are likely to vary in their admission and discharge practices. The rise in in-patient suicide rates over recent decades and an observed inverse relationship between average length of inpatient stay and in-patient suicide rates $^{1}$ both point to the importance of selection in explaining high rates of in-patient suicide.

Implicit in the belief that high rates of in-patient suicide are a result of a selection process is an acknowledgment that stays in hospital do not protect every patient from suicide. Variation in inpatient suicide rates might also be explained by differences in the extent to which different wards protect patients from suicide. Differences in the therapeutic milieu, the built environment, levels of observation, containment policies and staffing ratios might all alter in-patient suicide rates. This hypothesis is supported by a fall in in-patient suicide rates in the UK between 1997 and 2007 that was associated with measures to improve ward safety. ${ }^{5}$

There can be little doubt about the role of patient selection and subsequent failures of protection in in-patient suicide rates. However, these two factors might not be the only explanations for the high and variable rates of in-patient suicide. In previous papers, 
we have considered whether factors that can be part of the experience of admission to hospital, and that are known to be associated with suicide in the general community, might also be associated with some in-patient suicides. These factors include trauma, stigma, despair and loss of social role. If some in-patient suicides are associated with these factors, they could be labelled nosocomial suicides. ${ }^{6,7}$ Differences between hospitals in the extent to which they cause trauma, stigma, despair and loss of social role might be an under-recognised, but important, third factor explaining the extent and variation in in-patient suicide rates.

That some suicides might result from the inability of hospitals to prevent suicide whereas others might be due to elements intrinsic in the hospital care has parallels in other areas of medicine. For example, intensive care units prevent many deaths, but cannot prevent all; and in some instances, factors associated with intensive care itself, such as hospital-acquired infections, will cause some deaths that might otherwise not have occurred. ${ }^{8}$ Although it is well known that microorganisms endemic to hospitals can cause serious infection and even death, it has rarely been considered that adverse factors associated with psychiatric hospitalisation might result in suicide.

In general hospitals, the word 'nosocomial' is usually used to describe a causal association between an organism and a hospitalacquired infection. Here we use the term to describe suicides that would not have occurred had the patient not been admitted to a psychiatric hospital. In both instances, the term nosocomial has the caveat that it does not imply that being in hospital is the single cause of the adverse outcome. In relation to infection, host factors (such as age and debility) and non-hospital treatment factors (such as the previous use of antibiotics or current use of immunosuppressive medication) might also be important causes of a nosocomial infection. In relation to nosocomial suicide, we acknowledge that no suicide should be considered to be the result of any single factor and that host factors are likely to be very relevant. For example, it is very likely that the patient's perception of trauma, stigma, despair and loss of social role is what is most relevant and that the patient's perception is likely to be influenced by their presenting illness and vulnerabilities.

\section{Do observational data support the concept of nosocomial suicide?}

Half a century ago, in a landmark article - 'The Environment and Disease: Association or Causation?' - the English epidemiologist and statistician Austin Bradford Hill laid out guidelines as to how to proceed when there is an observed relationship between an environmental factor and disease that is 'clear cut, and beyond what we would care to attribute to the play of chance' and when experimental evidence was lacking or not practicable to obtain. ${ }^{9}$ Using what was learned from studies of the association between tobacco smoking and lung cancer, Hill proposed nine criteria that might influence belief in a causal association over a non-causal association. We chose to examine the concept of nosocomial suicide using Hill's criteria primarily because of their historic role in highlighting potential risk factors for closer examination with respect to causality. Although we acknowledge that modern statistical methods can be used to generate stronger causal inferences than Hill's criteria allow, ${ }^{10}$ databases examining the suicide trajectory of admitted and not admitted patients are few and the experimental evidence in relation to admission to hospital and suicide is slight.

Consideration of Hill's criteria cannot prove that a proportion of in-patient suicides are nosocomial; Hill did not consider the nine criteria to be 'hard-and-fast rules of evidence'. He did not think that any single criterion was proof of causality, nor that failure to fulfil any criterion was indisputable evidence against causation. Instead, he regarded the criteria as an aid to balancing the evidence to 'help us to make up our minds on the fundamental question - is there any other way of explaining the set of facts before us, and is there any other answer equally, or more likely, than cause and effect?'

\section{Method}

Here, we utilise Hill's criteria to weigh how seriously we should take the possibility that there might be a causal association between psychiatric hospitalisation and suicide. Hill's criteria are strength, consistency, specificity, temporality, biological gradient or dose response, plausibility, coherence, experiment and analogy.

\section{Results}

\section{Strength}

Hill considered that a causal association was more likely when those exposed to an environmental factor were much more likely to experience the disease than those not exposed. Hill did not specify what would constitute a strong association, but the examples he used - lung cancer among smokers and scrotal cancer among chimney sweeps - had odds ratios of 10-30 and 200 respectively. The association between psychiatric hospitalisation and in-patient suicide is statistically stronger than the association between smoking and lung cancer. Rates of in-patient suicide of over 600 per 100000 per annum found in modern psychiatric wards ${ }^{1}$ are 40 to 120 times higher than annual rates of suicide in the general community of between 5 and 15 per $100000 .{ }^{11}$ A strong association persists when non-admitted psychiatric patients, rather than the general community, are considered to be the control group. Rates of suicide among admitted patients are significantly higher than never-admitted patients with similar demographics and psychiatric disorders. ${ }^{12}$

\section{Consistency}

Hill considered that a causal association was more likely when an association was repeatedly observed by different researchers, in different places and in different circumstances. Acknowledging that there is very marked variation in suicide rates described in different studies, there is a consistent association between in-patient care and suicide, because rates of in-patient suicide are similarly high in studies from Australasia, Continental Europe, Nordic countries, the UK and the USA. ${ }^{1}$

\section{Specificity}

Hill considered that a stronger argument in favour of causation could be made if those exposed to an environmental factor had mortality because of the specific disease, but were not at increased risk of other forms of dying. Although suicide is probably the most common cause of death in psychiatric hospitals, the qualitative difference between suicide and other causes of death suggests this criterion might be less relevant.

\section{Temporality}

Although it is straightforwardly the case that gaining in-patient status comes before in-patient suicide, other temporal aspects are relevant. Although suicidal ideas and suicidal behaviour commonly precede admission to hospital and suicide, a meta-analysis found that the majority of people who completed suicide while they were in-patients had not expressed suicidal ideation at the point of admission and were not admitted after suicide attempts. ${ }^{13}$ In this sense, some suicidal behaviour is temporally related to psychiatric hospitalisation. Moreover, the very high rate of suicide immediately after discharge from hospital ${ }^{14,15}$ may also represent evidence of a temporal relationship between being in hospital and suicide. ${ }^{16}$ 


\section{Biological gradient}

Hill considered that belief in a causal association was strengthened by a 'dose-response curve'. By this he meant that hypotheses of causality were consistent with increased rates of disease in those who incurred a greater dose or exposure to the environmental factor. Meta-analysis suggests that in-patients with a greater number of previous admissions to hospital and with longer stays in hospital are at an increased risk of in-patient suicide compared with patients with fewer admissions and shorter admissions. ${ }^{13}$ Although longer admissions might also be associated with more suicides because of the increased time at risk and patients with more risk factors for suicide might have longer admissions, neither of these possibilities excludes a causal dose-related association between being in hospital and suicide.

\section{Plausibility}

Hill considered that a causal association was more likely if there was an understandable mechanism by which the environmental factor might be linked to disease. There is a plethora of literature about the adverse psychosocial consequences of in-patient psychiatric care. These adversities include humiliation, ${ }^{17}$ stigmatisation, ${ }^{18}$ loss of autonomy, ${ }^{19}$ perceived trauma ${ }^{20}$ and exposure to actual violence. ${ }^{21}$ Psychiatric in-patients can also experience a loss of accommodation and employment, and losses in social support and social role. ${ }^{22}$ It is known that negative emotional states, stigma ${ }^{23}$ and trauma ${ }^{24}$ are associated with suicide in the wider community, and there is no reason to think this is not the case in in-patient settings. In fact, it is likely that already vulnerable patients are more susceptible to the adversity associated with in-patient care. ${ }^{25}$ Variation in the type and extent of adversity between hospitals might also explain some of the variation in in-patient suicide rates.

\section{Coherence}

Hill considered that a causal mechanism should not 'seriously conflict with the generally known facts of the natural history $\ldots$ of the disease'. Situational suicide is a well-known phenomenon referring to self-destruction in a variety of adverse social circumstances. ${ }^{26}$ Moreover, suicide rates are elevated in custodial settings such as prisons ${ }^{27}$ and immigration detention centres. ${ }^{28}$ There is no incoherence in the notion that admission to hospital might cause some suicides.

\section{Experiment}

Hill considered that experimental or 'semi-experimental' evidence might impact one's belief with regard to causation. He gave examples of a change in the degree of exposure resulting in a change in the incidence of the disease. Psychiatric deinstitutionalisation might be regarded as a 'semi-experiment' for our purposes. Here the results of the relevant studies are conflicting, some finding psychiatric bed closures were associated with reduction in suicide, ${ }^{29}$ including in-patient suicides, ${ }^{30}$ some finding possible increases in suicide ${ }^{31}$ and some finding no change in suicide rates. ${ }^{32}$

\section{Analogy}

Hill considered that weaker evidence could be more readily accepted if there was a similar type of causal association that had been unequivocally accepted. Although analogy is a weaker form of argument than argument from specifics, the comparison between suicides in psychiatric hospitals and hospital-acquired illness, including infection, surgical accidents and medication errors, is obvious. In these examples, elements of the stay in hospital contribute substantially to morbidity and mortality.

\section{Discussion}

The relationship between psychiatric hospitalisation and suicide fulfils five of Hill's criteria - strength of association, consistency, plausibility, coherence and analogy. A further three criteria - temporality, biological gradient and experiment - are partially met. On weighing these results, the possibility that nosocomial suicides account for a proportion of in-patient suicides is worthy of serious consideration. But what more can be done to investigate the potential relationship between admission to hospital and suicide and what, if anything, should be done while we await the results of these investigations?

To date, only two experimental studies have examined the effects of any form of hospital admission on suicide outcomes. Neither study had sufficient sample size to examine whether admission to a hospital saves lives or causes suicide. Waterhouse \& Platt conducted a small randomised controlled trial (RCT) of admission to a general hospital $v$. community care after suicide attempt and found no difference in a variety of outcomes. ${ }^{33}$ Van de Sande and colleagues conducted a larger RCT comparing brief admission to a suicide crisis intervention unit with problemsolving aftercare $v$. care as usual. They also found no differences in a variety of outcomes. ${ }^{34}$

The two RCTs of hospitalisation $v$. out-patient management $^{33,34}$ suggest that a larger study with suicide as the main outcome measure might be possible. However, such a study would face very significant practical and ethical difficulties. The single biggest methodological issue would be the very large number of people that would need to be randomised. Compounding the need for a very large sample size are practical and ethical difficulties of recruiting patients for randomisation, because it is inevitable that, at any given crisis point, patients and clinicians will have preferences for either hospital or community treatment.

If a large-scale RCT could be performed, it would be a very direct test of whether in-patient psychiatric care caused more suicides than it prevented. A finding that admission to hospital was associated with a lower suicide mortality would not totally preclude the possibility that a smaller number of suicides result from the adverse effects of being in hospital but it would be very reassuring. Excess mortality in the hospitalisation arm would be alarming and would suggest that nosocomial suicides account for an important proportion of in-patient suicides.

In the final paragraphs of his article, under the heading 'The Case for Action', Hill argued that in deciding whether a known association could represent causation, we should 'have to consider what flows from that decision'. Whether or not to take action on a suspected causal link would, he said, depend heavily, not only on the strength of the evidence, but also on the costs of reducing exposure and the impact of disease to be prevented. Even if we only have a strong suspicion that some in-patient suicides are a direct result of psychiatric hospitalisation, it must be the case that the costs of some efforts to reduce exposure are so low, and the cost and impact of suicide are so high that we should take some action now.

Perhaps the most obvious step would be to pay more attention to the adverse aspects of being in hospital from the patient's perspective. Perceptions of trauma, stigma and loss of social support could easily be measured using self-report at the time of discharge. This sort of administrative clinical data would serve as a driver for improvements in hospital care and might provide important clinical information, potentially assisting some patients who have found hospital care unhelpful. Concerns about nosocomial suicide should also lead us to seek ways of admitting fewer patients, especially those patients who might find hospital treatment unacceptable. We should make greater efforts to maintain and increase resources for treatment in the community and discover ways to treat patients 
consensually and in their own homes rather than involuntarily in hospital. When admission to hospital is unavoidable, as it will often be, we must maximise the therapeutic aspects of in-patient psychiatric care and develop suicide-specific prevention strategies. ${ }^{35}$ We should do whatever possible to make in-patient care less traumatic and less stigmatising, and we should try to ensure that patients maintain social roles and social supports during their stay.

We acknowledge that there might be costs associated with admitting fewer patients, not least because treating patients safely in their own homes might be more expensive than admitting them. There might also be costs in measures to improve the therapeutic elements of hospital admission and in reducing its adverse aspects. However, we believe that the weight of arguments is sufficiently strong to support some measures to reduce nosocomial suicide.

Hill warned that that one should not advocate for change unless there is strong evidence, but he also emphasised that 'this does not imply crossing every "t," and [crossing] swords with every critic, before we act'. We agree. Although continued investigation into the impact of nosocomial suicide is important, we need not be certain that adverse factors associated with being in hospital contribute to the high rate of in-patient suicide before we take steps that might ameliorate them.

Matthew Michael Large, DMedSci FRANZCP, School of Psychiatry, University of New South Wales, Sydney, New South Wales, Australia; Daniel Thomas Chung, MD (candidate), Faculty of Medicine, University of New South Wales, Sydney, New South Wales, Australia; Michael Davidson, MD, University of Nicosia Medical School, University of Nicosia, Nicosia, Cyprus; Mark Weiser, MD, Department of Psychiatry, Sheba Medical Center, Tel Hashomer, Israel; Sackler School of Medicine, Tel Aviv University, Tel Aviv, Israel; Christopher James Ryan, MBBS, MHL, FRANZCP. Discipline of Psychiatry and the Centre for Values, Ethics and the Law in Medicine, University of Sydney, Sydney, New South Wales, Australia

Correspondence: Matthew Michael Large, Mental Health Services, The Prince of Wales Hospitals, Barker Street, Randwick, NSW 2031, Australia. Email: mmclarge@gmail. com

First received 8 Nov 2016, final revision 25 Feb 2017, accepted 27 Mar 2017

\section{References}

1 Walsh G, Sara G, Ryan CJ, Large M. Meta-analysis of suicide rates among psychiatric in-patients. Acta Psychiatr Scand 2015; 131: 174-84.

2 World Health Organization. Suicide Data. WHO, 2017. Available at http://www who.int/mental_health/prevention/suicide/suicideprevent/en/

3 Bassett D, Tsourtos G. Inpatient suicide in a general hospital psychiatric unit. A consequence of inadequate resources? Gen Hospital Psychiatry 1993; 15: 301-6.

4 Copas JB, Robin A. Suicide in psychiatric in-patients. Br J Psychiatry 1982; 141: 503-11.

5 Kapur N, Hunt IM, Windfuhr K, Rodway C, Webb R, Rahman MS, et al. Psychiatric in-patient care and suicide in England, 1997 to 2008: a longitudinal study. Psychol Med 2013; 43: 61-71.

6 Large M, Ryan C, Walsh G, Stein-Parbury J, Patfield M. Nosocomial suicide. Australas Psychiatry 2014; 22: 118-21.

7 Large MM, Ryan CJ. Disturbing findings about the risk of suicide and psychiatric hospitals. Soc Psychiatry Psychiatr Epidemiol 2014; 49: 1353-5.

8 Reunes S, Rombaut V, Vogelaers D, Brusselaers N, Lizy C, Cankurtaran M, et al. Risk factors and mortality for nosocomial bloodstream infections in elderly patients. Eur J Intern Med 2011; 22: e39-44.

9 Hill AB. The environment and disease: association or causation? Proc $R$ Soc Med 1965; 58: 295-300.

10 Höfler M. The Bradford Hill considerations on causality: a counterfactual perspective. Emerg Themes Epidemiol 2005; 2: 11

11 Varnik P. Suicide in the world. Int J Environ Res Public Health 2012; 9: 760-71.
12 Hjorthoj CR, Madsen T, Agerbo E, Nordentoft M. Risk of suicide according to level of psychiatric treatment: a nationwide nested case-control study. SOC Psychiatry Psychiatr Epidemiol 2014; 49: 1357-65.

13 Large M, Smith G, Sharma S, Nielssen O, Singh SP. Systematic review and meta-analysis of the clinical factors associated with the suicide of psychiatric in-patients. Acta Psychiatr Scand 2011; 124: 18-29.

14 Meehan J, Kapur $\mathrm{N}$, Hunt IM, Turnbull $\mathrm{P}$, Robinson J, Bickley $\mathrm{H}$, et al. Suicide in mental health in-patients and within 3 months of discharge. National clinical survey. Br J Psychiatry 2006; 188: 129-34.

15 Qin P, Nordentoft M. Suicide risk in relation to psychiatric hospitalization: evidence based on Iongitudinal registers. Arch Gen Psychiatry 2005; 62: 427-32

16 Chung DT, Ryan CJ, Large MM. Adverse experiences in psychiatric hospitals might be the cause of some postdischarge suicides. Bull Menninger Clin 2016; 80: $371-5$.

17 Svindseth MF, Dahl AA, Hatling T. Patients' experience of humiliation in the admission process to acute psychiatric wards. Nord J Psychiatry 2007; 61: 47-53.

18 Moses T. Stigma apprehension among adolescents discharged from brief psychiatric hospitalization. J Nerv Ment Dis 2011; 199: 778-89.

19 Sheehan KA, Burns T. Perceived coercion and the therapeutic relationship: a neglected association? Psychiatr Serv 2011; 62: 471-6.

20 Paksarian D, Mojtabai R, Kotov R, Cullen B, Nugent KL, Bromet EJ. Perceived trauma during hospitalization and treatment participation among individuals with psychotic disorders. Psychiatr Serv 2014; 65: 266-9.

21 Iozzino L, Ferrari $C$, Large $M$, Nielssen $O$, de Girolamo G. Prevalence and risk factors of violence by psychiatric acute inpatients: a systematic review and meta-analysis. PLOS One 2015; 10: e0128536.

22 Kinard EM, Klerman LV. Changes in life style following mental hospitalization. J Nerv Ment Dis 1980; 168: 666-72.

23 Schomerus G, Evans-Lacko S, Rusch N, Mojtabai R, Angermeyer MC, Thornicroft G. Collective levels of stigma and national suicide rates in 25 European countries. Epidemiol Psychiatr Sci 2015; 24: 166-71.

24 Panagioti M, Gooding PA, Tarrier N. Hopelessness, defeat, and entrapment in posttraumatic stress disorder: their association with suicidal behavior and severity of depression. J Nerv Ment Dis 2012; 200: 676-83.

25 Gallop R, McCay E, Guha M, Khan P. The experience of hospitalization and restraint of women who have a history of childhood sexual abuse. Health Care Women Int 1999; 20: 401-16.

26 Pridmore S, Auchincloss S, Walter G. Predicament suicide: an update. Australas Psychiatry 2015; 23: 411-4.

27 O'Driscoll C, Samuels A, Zacka M. Suicide in New South Wales Prisons, 1995 2005: towards a better understanding. Aust N Z J Psychiatry 2007; 41: 519-24.

28 Procter NG, De Leo D, Newman L. Suicide and self-harm prevention for people in immigration detention. Med J Aust 2013; 199: 730-2.

29 Pirkola S, Sohlman B, Heila $\mathrm{H}$, Wahlbeck K. Reductions in postdischarge suicide after deinstitutionalization and decentralization: a nationwide register study in Finland. Psychiatr Serv 2007; 58: 221-6.

30 Levi L, Werbeloff $N$, Pugachova I, Yoffe R, Large $M$, Davidson $M$, et al. Has deinstitutionalization affected inpatient suicide? Psychiatric inpatient suicide rates between 1990 and 2013 in Israel. Schizophr Res 2016; 173: 75-8.

31 Yoon J, Bruckner TA. Does deinstitutionalization increase suicide? Health Serv Res 2009; 44: 1385-405.

32 Johannessen $\mathrm{HA}$, Dieserud $\mathrm{G}$, Jakhelln $\mathrm{F}$, Zahl $\mathrm{PH}$, De Leo $\mathrm{D}$. Changes in institutional psychiatric care and suicidal behaviour: a follow-up study of inpatient suicide attempters in Baerum, Norway. Soc Psychiatry Psychiatr Epidemiol 2009; 44: $845-51$.

33 Waterhouse J, Platt S. General hospital admission in the management of parasuicide. A randomised controlled trial. Br J Psychiatry 1990; 156: 236-42.

34 van der Sande R, van Rooijen L, Buskens E, Allart E, Hawton K, van der Graaf $\mathrm{Y}$, et al. Intensive in-patient and community intervention versus routine care after attempted suicide. A randomised controlled intervention study. Br J Psychiatry 1997; 171: 35-41.

35 Ellis TE, Rufino KA, Allen JG, Fowler JC, Jobes DA. Impact of a suicide-specific intervention within inpatient psychiatric care: The Collaborative Assessment and Management of Suicidality. Suicide Life Threat Behav 2015. 12 January, doi: 10.1111/sltb.12151 (Epub ahead of print). 